\title{
Pairing and spectral statistics of low energy levels
}

\author{
R.A. Molina ${ }^{a}$ \\ Departamento de Física Atómica, Molecular y Nuclear, Universidad Complutense de Madrid, E-28040 Madrid, Spain and \\ Max-Planck-Institute für Physik komplexer Systeme, D-01187 Dresden, Germany
}

Received: 5 September 2005 / Revised: 12 December 2005 /

Published online: 16 June 2006 - (C) Società Italiana di Fisica / Springer-Verlag 2006

Communicated by A. Molinari

\begin{abstract}
The interplay of pairing with the different terms of the residual interaction is explored for the low-lying energy levels of semi-magic nuclei from the point of view of spectral statistics. The reduced degree of chaoticity present in this nuclei is shown to be directly related to the strength of the pairing interaction with respect to the total residual interaction. On the contrary, the pairing strength does not influence the spectral statistics near the ground state for nuclei with both neutrons and protons outside closed shells.
\end{abstract}

PACS. 21.60.Cs Shell model - 24.60.Lz Chaos in nuclear systems

\section{Introduction}

The behaviour of the energy level fluctuations is the fundamental signature of quantum chaos $[1-3]$. When the system is chaotic and has time reversal symmetry, its spectral statistics are the same as the ones for the GOE ensemble of random matrices. The energy levels are correlated and present level repulsion. On the other hand, the spectra of regular systems behave as an uncorrelated sequence of numbers and have Poisson statistics [4].

The atomic nucleus can be considered as a small laboratory where the interplay of regular and chaotic dynamics can be studied in complex many-body systems. One of the most important questions here is how the different parts of the nuclear interaction influence the statistical properties of the spectra [5]. Experimentally, the situation is rather clear above the one-nucleon emission threshold. A perfect agreement with GOE predictions has been obtained using sequences of levels measured by neutron resonance spectroscopy $[6,7]$. In the low-energy domain it is rather difficult to get large enough pure sequences. For this reason, even if the data coming from different nuclei are conveniently scaled and mixed, the conclusions are less clear [8-10]. As far as we know, there are only three cases where an experimental statistical analysis of a single nucleus has been done in the low-energy region: ${ }^{26} \mathrm{Al}$, ${ }^{116} \mathrm{Sn}$, and ${ }^{30} \mathrm{P}$. In all cases the results gave a behaviour intermediate between GOE and Poisson [11-13]. In the case of ${ }^{26} \mathrm{Al}$ a mechanism taking into account the effect of breaking the isospin symmetry has been proposed [14]. The same mechanism should hold for ${ }^{30} \mathrm{P}$. To the best of our knowledge, no explanation has been proposed for this non-universal behaviour in the case of ${ }^{116} \mathrm{Sn}$.

\footnotetext{
a e-mail: molina@mpipks-dresden.mpg.de
}

The Nuclear Shell Model with the effective two-body interaction in a restricted Hilbert space is the best available theoretical tool for calculating the properties of the low-lying states. Systematic studies for the spectral statistics using the Shell Model have been made in the $s d$ shell [15] and in the $p f$ shell [16]. The results in the $s d$ shell showed a general agreement with the Random Matrix Theory [15]. However, exceptions were found in the spectral statistics of nuclei in the $p f$ shell region [16-18] and in the $\mathrm{Pb}$ region $[19,20]$. The dynamics in the lowenergy region of semi-magic nuclei in these zones of the nuclear chart were close to regularity. The difference in the intensity of the $T=1$ and the $T=0$ residual interaction was considered to be the main responsible for that behaviour [16]. The $T=1$ residual interaction, the only part that is active on semi-magic nuclei, is not strong enough to perturb completely the regular motion in the mean field.

In this work, we want to study these questions by changing the intensity of the pairing interaction with respect to the realistic residual interaction and seeing how this affects the spectral statistics of nuclei. Our results show that the pairing interaction controls the spectral statistics of low-lying levels. Varying the strength of the pairing interaction, roughly from twice its value in the realistic force to almost zero, changes substantially the fluctuation properties of low-energy levels. We have found that an increase in the intensity of the pairing interaction drives the statistical properties of the spectra of low-lying states closer to Poisson. This means that the dynamics is more regular in this region. When we reduce the pairing interaction of the realistic force, the spectral statistical properties are more similar to the GOE properties. The dynamics in this case is more chaotic. The actual value of 
the increment or decrement of the level repulsion was very dependent on the intensity of the mean field. This can be shown comparing results for nuclei ${ }^{52} \mathrm{Ca}$ and ${ }^{48} \mathrm{Ca}$ where the residual interaction is exactly the same but the mean field is quite different. On the other hand, the spectral statistics in the high-energy region are essentially independent of the pairing strength. Some preliminary results of this study were already published in [21].

\section{Pairing interaction}

The pairing interaction is one of the main parts of realistic nuclear Hamiltonians. It is defined as

$H_{\text {par }}=$
$-G \sum_{j, j^{\prime}} \sum_{m, m^{\prime}>0}(-1)^{j+m}(-1)^{j^{\prime}+m^{\prime}} a_{j, m}^{+} a_{j,-m}^{+} a_{j^{\prime},-m^{\prime}} a_{j^{\prime}, m^{\prime}}$

where $j$ and $m$ are the angular-momentum quantum number and its third component for the single-particle orbits. The interaction scatters a pair of nucleon in the states $(j, m)(j,-m)$, that are coupled to total angular momentum $J^{\pi}=0^{+}$(so their total isospin is $T=1$ ), to any other state $\left(j^{\prime}, m^{\prime}\right)\left(j^{\prime},-m^{\prime}\right)$ with constant intensity $G$ independent of $j$ and $m$. The interested reader can consult a recent review about the importance of the pairing interaction in nuclear systems [22].

In the coupled scheme the pair-scattering matrix elements $\left\langle\left(j_{a}\right)^{2} ; J=0^{+}, T=1|V|\left(j_{c}\right)^{2} ; J=0^{+}, T=1\right\rangle$ of the pairing interaction have a very large value in realistic interactions due to the short-range attractive parts of the nucleon-nucleon potential. The low-lying states in the energy spectrum of even-even semi-magic nuclei are expected to be composed mainly of $J^{\pi}=0^{+}$-coupled pairs. It is meaningful to characterise the Shell-Model configurations of $n$ particles distributed over the $i$ orbitals by the number of particles combined in $0^{+}$pairs. For that purpose, the seniority quantum number $\nu$, defined as the number of particles not forming a $J=0$ pair, was introduced.

In order to investigate the effects of the pairing interaction in the statistical properties of the spectrum, we have used the Hamiltonian

$$
H=H_{\text {real }}+H_{\text {par }}(G),
$$

where $H_{\text {real }}$ is a realistic Hamiltonian. We have used the KB3 in the $p f$ shell [23] while in the region of $\mathrm{Pb}$ we have used an interaction derived from the Bonn-A potential by means of a $G$-matrix folded-diagram method by Covello and coworkers [24].

\section{Signatures of chaos in low-energy spectra as a function of the pairing strength}

The main statistic used in quantum chaos studies to characterise the spectrum is the nearest-neighbour spacing distribution $P(s)$. It is defined using the spacings between the

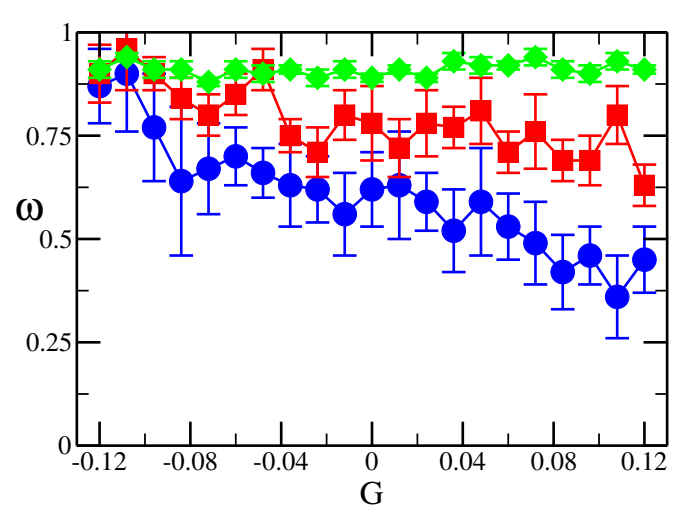

Fig. 1. The $\omega$ parameter for ${ }^{48} \mathrm{Ca}$ adding and subtracting some pairing interaction for different energy ranges. The circles show results for $E<5 \mathrm{MeV}$. The squares are the results for $E<10 \mathrm{MeV}$ and the diamonds for the complete spectra. The error bars represent the errors in the least-square fit to the Brody distribution.

unfolded levels $s_{i}=\epsilon_{i+1}-\epsilon_{i}$ with $\epsilon_{i}=\bar{N}\left(E_{i}\right)$, being $E_{i}$ the real energies of the system. The unfolding is needed to subtract the mean behaviour of the energy in order to do a proper comparison of the results with the Random Matrix Theory $[25,26]$. For the Shell Model matrices we have used an Edgeworth series to the fourth cumulant of the density [27] when all the spectrum was taken into account and the constant density formula [10] when we were in the low-energy region. The same unfolding methods were used in previous works and are explained more in detail in the corresponding refs. [16-18]. The unfolding is made for each value of the total angular momentum $J$, but all the spacings obtained up to a certain energy are put together to obtain better statistics.

We have fitted the $P(s)$ obtained with our Shell Model calculations to the Brody distribution formula [28],

$$
\begin{aligned}
& P(s, \omega)=\alpha(\omega+1) s^{\omega} \exp \left(-\alpha s^{\omega+1}\right), \\
& \alpha=\left(\Gamma\left[\frac{\omega+2}{\omega+1}\right]\right)^{\omega+1} .
\end{aligned}
$$

This distribution interpolates between the Poisson distribution $(\omega=0)$ of regular systems and the Wigner distribution $(\omega=1)$ of chaotic ones. The best fit to the GOE distribution being $\omega=0.95$. This parameter can be used as a simple quantitative measure of the degree of chaoticity.

The results for $\omega$ in the $p f$ shell $\left(0 f_{7 / 2}, 0 f_{5 / 2}, 1 p_{3 / 2}\right.$, $1 p_{1 / 2}$ orbits) are shown in fig. 1 , for the case of ${ }^{48} \mathrm{Ca}$, and in fig. 2, for the case of ${ }^{52} \mathrm{Ca}$. The number of spacings used in the fit is $N \approx 130$ for $E<5 \mathrm{MeV}, N \approx 650$ for $E<10 \mathrm{MeV}$ and $N \approx 12000$ in total, $E$ being the excitation energy with respect to the ground state of each nucleus. We can see the dramatic effect of pairing in the low-energy spectral fluctuations, while for the complete spectrum the effect cannot be seen at all. The minimum value of $G$ used, $G=-0.12 \mathrm{MeV}$, was chosen because it 


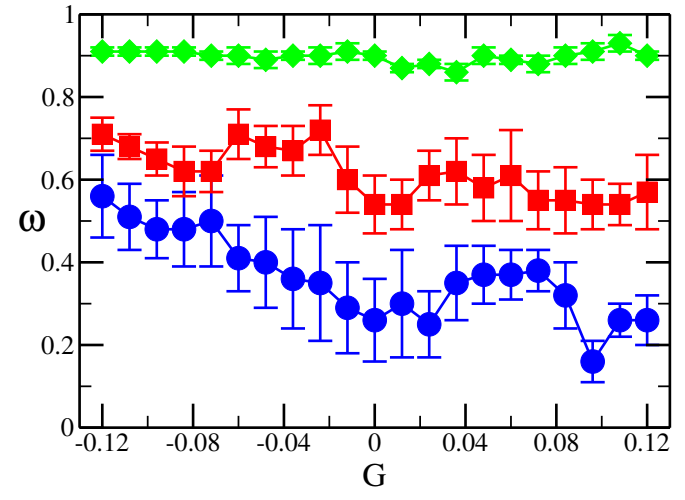

Fig. 2. The $\omega$ parameter for ${ }^{52} \mathrm{Ca}$ adding and subtracting some pairing interaction for different energy ranges. The circles show results for $E<5 \mathrm{MeV}$. The squares are the results for $E<10 \mathrm{MeV}$ and the diamonds for the complete spectra.

is a reasonable estimation of the intensity of the pairing force in the KB3 interaction. A least-square fit of the pairing matrix elements of KB3 gives a value of the pairing parameter of $G=0.117$. Therefore, subtracting a pairing of this intensity is almost like eliminating all the pairing in the realistic force while adding one is like doubling the strength of the pairing. We can see that, in the case of $E<5 \mathrm{MeV}$, the values of $\omega$ go from $\omega=0.87$, close to the GOE value, when we have no pairing, to $\omega=0.45$, when we have double pairing. For the case of the ${ }^{52} \mathrm{Ca}$ we go from $\omega=0.56$ to $\omega=0.26$. Although there are differences between the actual values of the Brody parameter, the behaviour as a function of $G$ is similar. We can explain this big difference between the values of $\omega$ between fig. 1 and fig. 2. For the case $G=0$, in which we take the realistic force without adding or subtracting pairing, the value of $\omega$ for ${ }^{52} \mathrm{Ca}$ is much lower than the one for ${ }^{48} \mathrm{Ca}$. In our model the particles are restricted to the four orbitals of the $p f$ shell and the two isotopes are related by a particle-hole transformation. The ${ }^{48} \mathrm{Ca}$ can be described as 8 particles moving in the $p f$ shell and the ${ }^{52} \mathrm{Ca}$ as 8 holes in the same shell. The particle-particle residual interaction is the same as the hole-hole one. The only difference between the two nuclei is due to the fact that in the case of ${ }^{48} \mathrm{Ca}$ the appropriate mean-field excitations are single-particle ones, while in the case of ${ }^{52} \mathrm{Ca}$ they are single-hole excitations. The gaps between the single-particle excitations in the $p f$ shell are smaller than the corresponding single-hole gaps and therefore the mean field acts strongly in the latter case. The gap between the particle orbits $f_{7 / 2}$ and $p_{3 / 2}$ is equal to about $2 \mathrm{MeV}$, while the gap between the hole orbits $\bar{f}_{7 / 2}$ and $\bar{p}_{3 / 2}$ is almost $4 \mathrm{MeV}[16]$. The parameter $\omega$ at low energy in semi-magic nuclei is given mainly by two effects, the amount of pairing interaction, and the relationship between the strength of the mean field and the strength of the residual interaction.

Pairing conserves the seniority quantum number. In the complete realistic interaction that is not true. But in the low-energy region pairing is most important and, although seniority is not fully conserved, it is approxi-

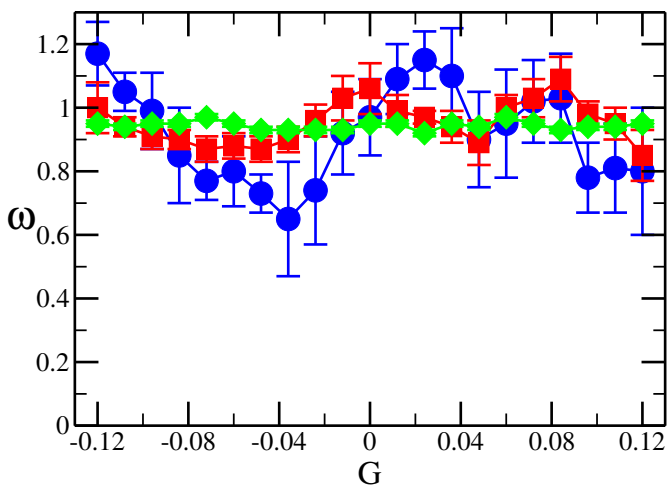

Fig. 3. The $\omega$ parameter for ${ }^{46} \mathrm{Sc}$ adding and subtracting some pairing interaction for different energy ranges. The circles show results for $E<5 \mathrm{MeV}$. The squares are the results for $E<$ $10 \mathrm{MeV}$ and the diamonds for the complete spectra.

mately so. In our analysis of the spacing distribution of the low-energy levels we are including states with different seniority. The level repulsion between them is reduced because of the partial conservation of the seniority quantum number and so is the value of $\omega$ and the chaoticity of the dynamics.

We have also made the same calculation changing the value of the pairing interaction with ${ }^{46} \mathrm{Sc}$ (fig. 3). We have only used the states with quantum numbers $J=0-12$ and $T=3$. The number of spacings used in the statistical analysis was $N=155$ for $E<5 \mathrm{MeV}, N=1328$ for $E<10 \mathrm{MeV}$, and $N=25998$ for the total spectrum. In this case the effects are not noticeable and the value of $\omega$ is always in the chaotic region. For $E<5 \mathrm{MeV}$ we can see oscillations in the value of $\omega$, but they are probably due to the small size of the statistical sample. In nuclei with both kind of particles, protons and neutrons, outside closed shells pairing is not so important. Both the $T=0$ and the $T=1$ residual interactions are working in this nucleus and they are strong enough to perturb completely the mean-field single-particle motion at all energies.

To show the generality of these effects we have also made calculations in the $\mathrm{Pb}$ region. The core is the nucleus of $\mathrm{Pb}^{212}$ with $Z=82$ and $N=126$. We have used a valence space with the orbits $1 g_{9 / 2}, 1 g_{7 / 2}, 2 d_{5 / 2}, 2 d_{3 / 2}$, $3 s_{1 / 2}, 0 j_{15 / 2}$ for neutrons (the only part needed in the different isotopes of $\mathrm{Pb}$ ). A maximum value of $G=0.08 \mathrm{MeV}$ was used. A least-squares fit of the pairing matrix elements gives a pairing force with $G=0.071$. In fig. 4 we see a similar behaviour for ${ }^{212} \mathrm{~Pb}$. In this particular case the value of $\omega$ for $G=0$ is very low. This is due to the strength of the mean field for heavy nuclei [19]. But the addition or the subtraction of pairing produces a decrease or an increase of the chaoticity of low-energy spectra much in the same way as in the $p f$ shell.

In both regions we have checked the effect of pairing in other statistics like $\Delta_{3}$ that measures long range correlations of the spectra [29] and the entropy localisation length that measures the localisation of the wave functions in the single particle basis [30] and we have found 


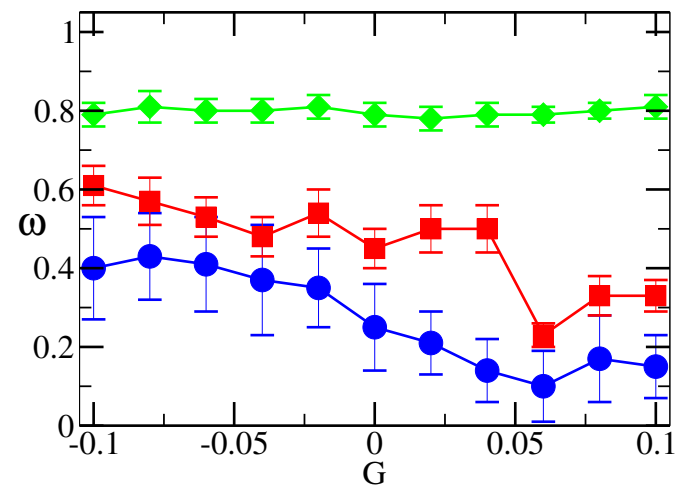

Fig. 4. The $\omega$ parameter for ${ }^{212} \mathrm{~Pb}$ adding and subtracting some pairing interaction for different energy ranges. The circles show results for $E<1 \mathrm{MeV}$ (108 levels). The squares are the results for $E<2 \mathrm{MeV}$ (407 levels) and the diamonds for the complete spectra (13973 levels).

no significant effects (not shown). In view of the previous results this was completely expected because both of these statistics refer to the whole spectra including all levels and we have seen that $\omega$ for the complete spectrum was not affected by pairing.

\section{Conclusions}

We have explored how the interplay between the pairing interaction and the residual interaction affects the spectral statistics of nuclei. We have shown that the pairing interaction is the main cause for the regular behaviour in spectral statistics of low-energy states of semi-magic nuclei. Adding and subtracting pairing from realistic interactions for nuclei in the $p f$ shell and in the $\mathrm{Pb}$ region we have seen how the value of the Brody parameter $\omega$, that measures the regular o chaotic behaviour of the spectra, is reduced or increased. We have also seen that the spectral statistics of the complete spectrum or the statistics of the wave functions are not modified substantially. This explains previous results for semi-magic nuclei. Partial conservation of seniority is proposed as the underlying mechanism for the effect of pairing in low energy spectra.

For ${ }^{116} \mathrm{Sn}$ the experimental result for the $\omega$ parameter according to [12] is $\omega=0.51 \pm 0.19$. This is a semi-magic nuclei with 16 neutrons outside closed shells. Although a calculation of enough levels for this nuclei is outside our present capabilities, this result can be explained by the same mechanism we have studied. It is also worth mentioning that the studied mechanism is not exclusive to the pairing force. Other integrable Hamiltonians can have similar effects, for example, $S U(3)$, as the effect seen here is related to the fact that some specific integrable part of the Hamiltonian is dominant at low energy.

The author would like to thank J.M.G. Gómez under whose supervision this work was started. It is a pleasure to specially acknowledge J. Retamosa for useful comments and discussions. This work was supported in part by the Spanish Government grant BFM2003-04147-C02-01.

\section{References}

1. O. Bohigas, M.J. Giannoni, C. Schmidt, Phys. Rev. Lett. 52, 1 (1984).

2. M.J. Giannoni, A. Voros, J. Zinn-Justin (Editors), Chaos and Quantum Physics (Les Houches Session LII, NorthHolland, 1989).

3. F. Haake, Quantum Signatures of Chaos (Springer-Verlag, Heidelberg, 2001).

4. M.V. Berry, Proc. R. Soc. London, Ser. A 400, 229 (1985).

5. A. Volya, V. Zelevinsky, B.A. Brown, Phys. Rev. C 65, 054312 (2002).

6. R.U. Haq, A. Pandey, O. Bohigas, Phys. Rev. Lett. 48, 1086 (1982).

7. O. Bohigas, H.A. Weidenmüller, Annu. Rev. Nucl. Part. Sci. 46, 421 (1988).

8. G.E. Mitchell, E.G. Bilpuch, P.M. Endt, J.F. Shriner jr., Phys. Rev. Lett. 61, 1473 (1988).

9. J.F. Shriner jr., E.G. Bilpuch, P.M. Endt, G.E. Mitchell, Z. Phys. A 335, 393 (1990).

10. J.F. Shriner jr, G.E. Mitchell, T. von Egidy, Z. Phys. A 338, 309 (1991).

11. J.F. Shriner jr., E.G. Bilpuch, P.M. Endt, G.E. Mitchell, Z. Phys. A 335, 393 (1990).

12. S. Raman et al., Phys. Rev. C 43, 521 (1991).

13. J.F. Shriner jr., C.A. Grossmann, G.E. Mitchell, Phys. Rev. C 62, 054305 (2000).

14. T. Guhr, H.A. Weidenmüller, Ann. Phys. (N.Y.) 199, 412 (1990).

15. V. Zelevinsky, B.A. Brown, N. Frazier, M. Horoi, Phys. Rep. 276, 85 (1996).

16. R.A. Molina, J.M.G. Gómez, J. Retamosa, Phys. Rev. C 63, 014311 (2001).

17. E. Caurier, J.M.G. Gómez, V.R. Manfredi, L. Salasnich, Phys. Lett. B 365, 7 (1996).

18. J.M.G. Gómez, V.R. Manfredi, L. Salasnich, E. Caurier, Phys. Rev. C 58, 2108 (1998).

19. M.S. Bae, T. Otsuka, T. Mizusaki, N. Fukunishi, Phys. Rev. Lett. 69, 2349 (1992).

20. R.A. Molina, to be published.

21. J.M.G. Gómez, R.A. Molina, J. Retamosa, in Proceedings of the 7th Spring Seminar on Nuclear Physics, Challenges on Nuclear Structure, edited by A. Covello (World Scientific, Singapore, 2002).

22. D.J. Dean, M. Hjorth-Jensen, Rev. Mod. Phys. 75, 607 (2003).

23. A. Poves, A.P. Zuker, Phys. Rep. 70, 235 (1981).

24. A. Covello, private communication.

25. T. Guhr, A. Müller-Groeling, H.A. Weidenmüller, Phys. Rep. 299, 189 (1998).

26. J.M.G. Gómez, R.A. Molina, A. Relaño, J. Retamosa, Phys. Rev. E 66, 036209 (2002).

27. S.S.M. Wong, Nuclear Statistical Spectroscopy (Oxford University Press, New York, 1986).

28. T.A. Brody, Lett. Nuovo Cimento 7, 482 (1973).

29. M.L. Mehta, F.J. Dyson, J. Math. Phys. 4, 713 (1963).

30. F.M. Izrailev, J. Phys. A 22, 865 (1989). 